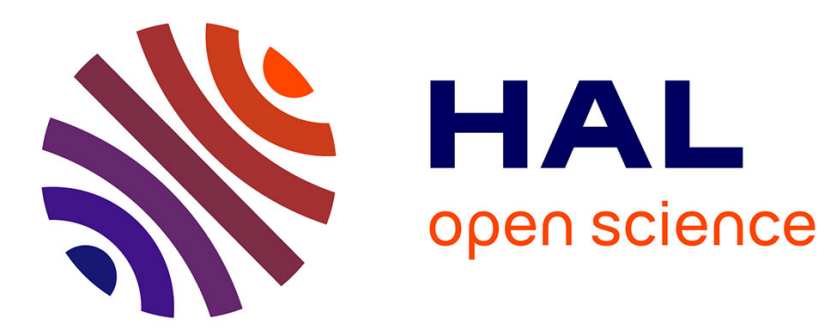

\title{
A sensitivity analysis of a class of semi-coercive variational inequalities using recession tools
}

\author{
Khalid Addi, Samir Adly, Daniel Goeleven, Hassan Saoud
}

\section{To cite this version:}

Khalid Addi, Samir Adly, Daniel Goeleven, Hassan Saoud. A sensitivity analysis of a class of semicoercive variational inequalities using recession tools. Journal of Global Optimization, 2008, 40 (1-3), pp.7-27. 10.1007/s10898-007-9207-4 . hal-00465588

\section{HAL Id: hal-00465588 \\ https://hal.science/hal-00465588}

Submitted on 18 Oct 2017

HAL is a multi-disciplinary open access archive for the deposit and dissemination of scientific research documents, whether they are published or not. The documents may come from teaching and research institutions in France or abroad, or from public or private research centers.
L'archive ouverte pluridisciplinaire HAL, est destinée au dépôt et à la diffusion de documents scientifiques de niveau recherche, publiés ou non, émanant des établissements d'enseignement et de recherche français ou étrangers, des laboratoires publics ou privés. 


\title{
A sensitivity analysis of a class of semi-coercive variational inequalities using recession tools
}

\author{
Khalid Addi · Samir Adly • Daniel Goeleven • \\ Hassan Saoud
}

\begin{abstract}
Using the recession analysis we study necessary and sufficient conditions for the existence and the stability of a finite semi-coercive variational inequality with respect to data perturbation. Some applications of the abstract results in mechanics and in electronic circuits involving devices like ideal diode and practical diode are discussed.
\end{abstract}

Keywords Finite variational inequalities $\cdot$ Recession analysis $\cdot$ Convex analysis $\cdot$ Positive semidefinite matrices - Clipping circuit - Ideal diode model · Practical diode model · coulomb friction

\section{Introduction and position of the problem}

The theory of variational inequalities, with its wide range of applications in engineering, economics, finance, industy and mechanics, has become a well-established and fruitful area of research. After the fundamental work of Lions and Stampacchia [18], this theory have been studied intensively. With the contributions of Brézis [7, 8], Duvaut Lions [10], Browder [9],

The research of S. Adly has been supported by the "Fondation EADS" and the ANR project "Guidage".

\author{
K. Addi · D. Goeleven \\ IREMIA, Université de La Réunion, \\ Saint-Denis 97400, France \\ e-mail: khalid.addi@univ-reunion.fr \\ D. Goeleven \\ e-mail: daniel.goeleven@univ-reunion.fr \\ S. Adly $(\varangle) \cdot$ H. Saoud \\ XLIM UMR-CNRS 6172, Université de Limoges, \\ Limoges 87060, France \\ e-mail: samir.adly@unilim.fr \\ H. Saoud \\ e-mail: hassan.saoud@etu.unilim.fr
}


Kinderlehrer and Stampacchia [16], Panagiotopoulos [19], Goeleven Motreanu [13] (among others), this field has known an increasing growth in both theory and applications. Several books and articles have documented the basic theory, the numerical approach and applications in applied science as well. This theory was used as a tool for the study of partial differential equations with applications essentially drawn from mechanics (Signorini problem, obstacle problems in elasticity, etc).

We consider the following finite dimensional variational inequality

$$
\operatorname{VI}(M, q, \Phi, K)\left\{\begin{array}{l}
\text { Find } u \in K \text { such that } \\
\langle M u+q, v-u\rangle+\Phi(v)-\Phi(u) \geq 0, \quad \forall v \in K
\end{array}\right.
$$

where $M \in \mathbb{R}^{n \times n}$ is a matrix, $q \in \mathbb{R}^{n}$ is a vector, $K$ is a nonempty closed convex set of $\mathbb{R}^{n}$ and $\Phi: \mathbb{R}^{n} \rightarrow \mathbb{R} \cup\{+\infty\}$ is a proper convex and lower semicontinuous function.

We denote by:

$$
\operatorname{Dom}(\Phi)=\left\{v \in \mathbb{R}^{n}: \Phi(v)<+\infty\right\},
$$

the effective domain of $\Phi$.

Let us now suppose that the assumptions $(\mathcal{H})$ described below are satisfied:

( $\left.H_{1}\right) \quad M \in \mathbb{R}^{n \times n}$ is a symmetric and positive semidefinite matrix;

$\left(H_{2}\right) \Phi: \mathbb{R}^{n} \rightarrow \mathbb{R} \cup\{+\infty\}$ is a proper, convex, lower semicontinuous and bounded from below;

$\left(H_{3}\right) \quad K \subset \mathbb{R}^{n}$ is a closed and nonempty convex set

$\left(H_{4}\right) \quad 0 \in \operatorname{Dom} \Phi \cap K$.

Several theoretical existence results for $\operatorname{VI}(M, q, \Phi, K)$ in general reflexive Banach spaces and governed by a general operator $M$ (not necessarily linear) are well known when a coerciveness condition hold for the operator $M$. We can cite for instance the contributions of Lions [17], Brézis [7,8], Browder [9] etc .... However, the variational formulation of many engineering problems leads generally to non-coercive variational inequalities (e.g., problems in mechanics which admits nontrivial virtual rigid body displacement). These problems are formulated by semi-coercive variational inequalities and was studied first by Fichera [12] and Lions and Stampacchia [18], Duvaut and Lions [10] (for problems with frictional type functionals). Recently many mathematicians and engineers has focused their attention on non-coercive unilateral problems, using several different approaches such as the critical point theory, the Leray-Schauder degree theory, the recession analysis or the regularization method by approximating non-coercive problems by coercive ones (see e.g., [1,4-6,21,22] and references cited therein). The main concern of these contributions is the obtainment of necessary or sufficient conditions for the solvability of such problems in a general setting by imposing some compacity conditions and some compatibility conditions on the right hand term $q$. More recently, Adly et al. [2,3] has considered the situations in which the existence of the solution is stable with respect to small uniform perturbations of the data of the problem. This result should be of great interest for problems in finance and engineering where the data are known only with a certain precision and it is desired that further refinement of the data should not cause the emptiness of the set of solutions.

Our aim is thus to characterize the sensitivity of $\mathrm{VI}(M, q, \Phi, K)$ with respect to the perturbations of the data $M, q, \Phi$ and $K$. In this paper, we only discuss the case of finite variational inequalities. Some applications of our main results in electronics and mechanics are given in Sect. 4. 


\section{Preliminaries and notations}

Let us first recall some background results from convex analysis which will be used later.

Let $K$ be a closed convex subset of $\mathbb{R}^{n}$, the recession cone of $K$ is the closed convex cone

$$
K_{\infty}:=\bigcap_{t>0}\left[\frac{K-x_{0}}{t}\right],
$$

with $x_{0}$ arbitrarily chosen in $K$.

We denote by $\Gamma_{0}\left(\mathbb{R}^{n}\right)$ the set of all proper, convex and lower semicontinuous extended real valued functions $\Phi: \mathbb{R}^{n} \rightarrow \mathbb{R} \cup\{+\infty\}$.

Let $\Phi \in \Gamma_{0}\left(\mathbb{R}^{n}\right)$ be given. The recession function $\Phi_{\infty}$ of $\Phi$ is defined by:

$$
\Phi_{\infty}(x):=\lim _{\lambda \rightarrow+\infty} \frac{\Phi\left(x_{0}+\lambda x\right)-\Phi\left(x_{0}\right)}{\lambda},
$$

where $x_{0} \in$ Dom $\Phi$ is an arbitrary element. We set

$$
\operatorname{ker} \Phi_{\infty}=\left\{x \in \mathbb{R}^{n}: \Phi_{\infty}(x)=0\right\},
$$

which is clearly a closed convex cone in $\mathbb{R}^{n}$.

The Fenchel conjugate $\Phi^{*}: \mathbb{R}^{n} \rightarrow \mathbb{R} \cup\{+\infty\}$ of $\Phi$ is defined by:

$$
\Phi^{*}\left(x^{*}\right)=\sup _{x \in \mathbb{R}^{n}}\left\{\left\langle x^{*}, x\right\rangle-\Phi(x)\right\} .
$$

We recall also that the convex subdifferential of $\Phi$ at a point $x \in$ Dom $\Phi$ is defined by:

$$
\partial \Phi(x)=\left\{w \in \mathbb{R}^{n}:\langle w, y-x\rangle \leq \Phi(y)-\Phi(x), \quad \forall y \in \mathbb{R}^{n}\right\} .
$$

The effective domain of the multivalued mapping $\partial \Phi$ is defined by

$$
\mathrm{D}(\partial \Phi)=\left\{x \in \mathbb{R}^{n}: \partial \Phi(x) \neq \emptyset\right\} .
$$

We note that

$$
\mathrm{D}(\partial \Phi) \subset \operatorname{Dom} \Phi
$$

The range of the multivalued mapping $\partial \Phi$ is defined by

$$
R(\partial \Phi)=\bigcup_{x \in \mathbb{R}^{n}} \partial \Phi(x)
$$

We have the following Fenchel correspondence:

$$
w \in \partial \Phi(x) \Longleftrightarrow x \in \partial \Phi^{*}(w) .
$$

Hence,

$$
R(\partial \Phi)=D\left(\partial \Phi^{*}\right) \subset \operatorname{Dom} \Phi^{*} .
$$

The indicator function to a convex set $K$ is given by:

$$
I_{K}(x)=\left\{\begin{array}{cl}
0 & \text { if } x \in K \\
+\infty & \text { if } x \notin K
\end{array}\right.
$$

If $K$ is a closed cone, its polar is defined by

$$
K^{\circ}=\left\{x^{*} \in \mathbb{R}^{n}:\left\langle x^{*}, x\right\rangle \leq 0, \quad \forall x \in K\right\} .
$$


The support function to $K$ is defined by:

$$
\sigma_{K}(w)=\left(I_{K}\right)^{*}(w)=\sup _{x \in K}\langle w, x\rangle .
$$

We recall that the barrier cone of $K$ is defined by:

$$
\mathcal{B}(K)=\left\{w \in \mathbb{R}^{n}: \sup _{x \in K}\langle w, x\rangle<+\infty\right\}=\text { Dom } \sigma_{K} .
$$

It is well-known that if $K$ is a non-empty closed and convex subset, then

$$
\mathcal{B}(K)^{\circ}=K_{\infty} .
$$

Therefore,

$$
\overline{\mathcal{B}(K)}=\left(K_{\infty}\right)^{\circ} .
$$

We recall that for $\Phi_{1}, \Phi_{2} \in \Gamma_{0}\left(\mathbb{R}^{n}\right)$, the infimal convolution (or the epigraphical sum) is defined by:

$$
\left(\Phi_{1} \square \Phi_{2}\right)(x)=\inf _{y+z=x}\left\{\Phi_{1}(y)+\Phi_{2}(z)\right\} .
$$

We say that the infimal convolution is exact provided that the infimum appearing in (6) is achieved.

We note that

$$
\operatorname{Dom}\left(\Phi_{1} \square \Phi_{2}\right)=\operatorname{Dom}\left(\Phi_{1}\right)+\operatorname{Dom}\left(\Phi_{2}\right) .
$$

We recall that if $\Phi_{1}$ (or $\Phi_{2}$ ) is continuous on $\mathbb{R}^{n}$, then

$$
\left(\Phi_{1}+\Phi_{2}\right)^{*}=\Phi_{1}^{*} \square \Phi_{2}^{*}
$$

and the infimal convolution $\Phi_{1}^{*} \square \Phi_{2}^{*}$ is exact.

Let us finally recall the following proposition

Proposition 1 Let $\Psi \in \Gamma_{0}\left(\mathbb{R}^{n}\right)$ and $p \in \mathbb{R}^{n}$ be given. We have:

(i) $p \in \overline{\operatorname{Dom} \Psi^{*}} \Longleftrightarrow \Psi_{\infty}(w) \geq\langle p, w\rangle, \quad \forall w \in \mathbb{R}^{n}$;

(ii) $p \in \operatorname{Int}\left(\operatorname{Dom} \Psi^{*}\right) \Longleftrightarrow \Psi_{\infty}(w)>\langle p, w\rangle, \quad \forall w \in \mathbb{R}^{n} \backslash\{0\}$.

Proof For a proof see Corollary 13.3.4 in [20].

\section{Characterization results}

The solutions set of $\operatorname{VI}(M, q, \Phi, K)$ will be denoted by

$\operatorname{Sol}(M, q, \Phi, K):=\{u \in K:\langle M u+q, v-u\rangle+\Phi(v)-\Phi(u) \geq 0, \quad \forall v \in K\}$.

The following resolvent set will also play an important role

$$
\mathcal{R}(M, \Phi, K)=\left\{-q \in \mathbb{R}^{n}: \operatorname{Sol}(M, q, \Phi, K) \neq \emptyset\right\} .
$$

Let us introduce the following function $\Psi: \mathbb{R}^{n} \rightarrow \mathbb{R} \cup\{+\infty\}$ defined by

$$
\Psi(u)=\frac{1}{2}\|Q u\|^{2}+\Phi(u)+I_{K}(u),
$$

where $Q=I-P_{\operatorname{ker}(M)}$ and $P_{\operatorname{ker}(M)}$ denotes the orthogonal projector from $\mathbb{R}^{n}$ to $\operatorname{ker}(M)$. 
Remark 1 We first remark that $\operatorname{VI}(M, q, \Phi, K)$ is equivalent to the following variational inclusion: find $u \in K$ such that

$$
0 \in M u+q+\partial\left(\Phi+I_{K}\right)(u),
$$

where $I_{K}$ denotes the indicator function of $K$. Hence,

$$
-q \in M u+\partial\left(\Phi+I_{K}\right)(u) \subset \bigcup_{u \in \mathbb{R}^{n}}\left(M u+\partial\left(\Phi+I_{K}\right)(u)\right) \subset R(M)+R\left(\partial\left(\Phi+I_{K}\right)\right) .
$$

Using (2), we get

$$
-q \in R(M)+\operatorname{Dom}\left(\Phi+I_{K}\right)^{*}
$$

We have the following lemma.

Lemma 1 Suppose that the assumptions $(\mathcal{H})$ hold. We have

$$
\operatorname{Dom} \Psi^{*}=R(M)+\operatorname{Dom}\left(\Phi+I_{K}\right)^{*} .
$$

Proof Let us note first that the function $\Psi$ defined in (9) can be rewritten

$$
\Psi(u)=\frac{1}{2}\left(\operatorname{dist}_{\mathrm{ker}(M)}(u)\right)^{2}+\left(\Phi+I_{K}\right)(u) .
$$

Using (8) for $\Phi_{1}=\frac{1}{2}\left(\operatorname{dist}_{U}(\cdot)\right)^{2}$ (which is convex and continuous) and $\Phi_{2}=\left(\Phi+I_{K}\right) \in$ $\Gamma_{0}\left(\mathbb{R}^{n}\right)$, we get

$$
\Psi^{*}=\left[\frac{1}{2} \operatorname{dist}_{\operatorname{ker}(M)}(\cdot)^{2}\right]^{*} \square\left[\Phi+I_{K}\right]^{*}
$$

Hence

$$
\operatorname{Dom} \Psi^{*}=\operatorname{Dom}\left[\frac{1}{2} \operatorname{dist}_{k e r(M)}(\cdot)^{2}\right]^{*}+\operatorname{Dom}\left[\Phi+I_{K}\right]^{*} .
$$

On the other hand, we have:

$$
\frac{1}{2} \operatorname{dist}_{\operatorname{ker}(M)}(\cdot)^{2}=\frac{1}{2}\|\cdot\|^{2} \square I_{\operatorname{ker}(M)} .
$$

Using (8) again, we obtain

$$
\left[\frac{1}{2} \operatorname{dist}_{\operatorname{ker}(M)}(\cdot)^{2}\right]^{*}=\frac{1}{2}\|\cdot\|^{2}+I_{\operatorname{ker}(M)^{\perp}} .
$$

Hence

$$
\operatorname{Dom}\left[\operatorname{dist}_{\operatorname{ker}(M)}(\cdot)^{2}\right]^{*}=\operatorname{ker}(M)^{\perp} \text {. }
$$

Consequently,

$$
\operatorname{Dom} \Psi^{*}=R(M)+\operatorname{Dom}\left[\Phi+I_{K}\right]^{*} .
$$

Proposition 2 Suppose that assumptions $(\mathcal{H})$ hold. Then a necessary condition for the existence of a solution of $\mathrm{VI}(M, q, \Phi, K)$ is that

$$
\langle q, w\rangle+\Phi_{\infty}(w) \geq 0, \quad \forall w \in \operatorname{ker}(M) \cap K_{\infty} .
$$


Proof Using Remark 1 and Lemma 1, we have

$$
\mathcal{R}(M, \Phi, K) \subset \overline{\operatorname{Dom}\left(\Psi^{*}\right)} .
$$

Using Part (i) of Proposition 1, we have

$$
\overline{\operatorname{Dom}\left(\Psi^{*}\right)}=\left\{g \in \mathbb{R}^{n}:\langle g, w\rangle \leq \Psi_{\infty}(w), \quad \forall w \in \mathbb{R}^{n}\right\} .
$$

It can also easily be checked that the recession function $\Psi_{\infty}$ associated to $\Psi$ in (9) is given by

$$
\Psi_{\infty}(w)=I_{\operatorname{ker}(M)}(w)+\Phi_{\infty}(w)+I_{K_{\infty}}(w) .
$$

Consequently if $\operatorname{Sol}(M, q, \Phi, K) \neq \emptyset$ then $-q \in \mathcal{R}(M, \Phi, K)$ and thus:

$$
\langle q, w\rangle+\Phi_{\infty}(w) \geq 0, \quad \forall w \in \operatorname{ker}(M) \cap K_{\infty}
$$

which competes the proof of Proposition 2.

Proposition 3 Let $C$ be a non-empty closed convex subet of $\mathbb{R}^{n}$. We have

$$
\text { Int } \mathcal{B}(C) \neq \emptyset \Longleftrightarrow C_{\infty} \cap\left(-C_{\infty}\right)=\{0\},
$$

where $\mathcal{B}(C)$ denotes the barrier cone of $C$ defined in (3).

Proof Since $\mathcal{B}(C)=\operatorname{Dom}\left(I_{C}\right)^{*}$, we have

$$
\text { Int } \mathcal{B}(C) \neq \emptyset \Longleftrightarrow \text { Int } \operatorname{Dom}\left(I_{C}\right)^{*} \neq \emptyset \text {. }
$$

Using Part (ii) of Proposition 1 for $\Psi=I_{C}$, we have

$$
\text { Int } \operatorname{Dom}\left(I_{C}\right)^{*}=\left\{g \in \mathbb{R}^{n}:\langle g, w\rangle<I_{C_{\infty}}(w), \quad \forall w \in \mathbb{R}^{n}, w \neq 0\right\} .
$$

Hence,

$$
\text { Int } \mathcal{B}(C)=\left\{g \in \mathbb{R}^{n}:\langle g, w\rangle<0, \forall w \in C_{\infty}, w \neq 0\right\} \text {. }
$$

Therefore,

$$
\operatorname{Int} \mathcal{B}(C)=\operatorname{Int}\left(C_{\infty}\right)^{\circ} .
$$

Consequently, if Int $\mathcal{B}(C) \neq \varnothing$ then there exists $g \in \mathbb{R}^{n}$ such that:

$$
\langle g, w\rangle<0, \quad \forall w \in C_{\infty}, \quad w \neq 0,
$$

which implies that necessarily $C_{\infty} \cap-C_{\infty}=\{0\}$.

Conversely, suppose that $C_{\infty} \cap-C_{\infty}=\{0\}$. Arguing by contradiction, let us suppose that Int $\left(C_{\infty}\right)^{\circ}=\emptyset$. Since $\left(C_{\infty}\right)^{\circ}$ is cone, then there exists a linear subspace $E$ of $\mathbb{R}^{n}$ with $0 \leq \operatorname{dim}_{\mathbb{R}}(E) \leq n-1$ such that: $\left(C_{\infty}\right)^{\circ} \subset E$ (see e.g. [14], p. 33). Therefore, $E^{\perp} \subset\left(C_{\infty}\right)^{\circ \circ}=C_{\infty}$, where $E^{\perp}$ is the orthogonal of $E$. This contradicts the assumption $C_{\infty} \cap-C_{\infty}=\{0\}$ (since $\operatorname{dim}_{\mathbb{R}} E^{\perp} \geq 1$ and $E^{\perp} \subset C_{\infty}$ ). Hence, Int $\left(C_{\infty}\right)^{\circ} \neq \emptyset$. Consequently, Int $\mathcal{B}(C) \neq \emptyset$, which completes the proof of Proposition 3.

Remark 2 A subset $C$ satisfying the condition Int $\mathcal{B}(C) \neq \emptyset$ is called well-positioned (see Proposition 2.1 [2]). An other characterization of this class of convex subsets in infinite dimensional spaces is also given in Lemma 2.4 [2] and Proposition 2.1 [3]. Note that in finite dimensional spaces every subset which contains no line is well-positioned. 
Definition 1 The set defined by

$$
\left\{q \in \mathbb{R}^{n}:\langle q, w\rangle+\Phi_{\infty}(w)>0, \quad \forall w \in \operatorname{ker}(M) \cap K_{\infty} \backslash\{0\}\right\},
$$

is called the compatibility set of the variational inequality $\operatorname{VI}(M, q, \Phi, K)$.

The following proposition shows that the topological interior of the resolvent set coincides with the compatibility set of the variational inequality $\operatorname{VI}(M, q, \Phi, K)$.

Proposition 4 Suppose that assumptions $(\mathcal{H})$ hold. We have

$$
\operatorname{Int} \mathcal{R}(M, \Phi, K)=\left\{q \in \mathbb{R}^{n}:\langle q, w\rangle+\Phi_{\infty}(w)>0, \quad \forall w \in \operatorname{ker}(M) \cap K_{\infty} \backslash\{0\}\right\} .
$$

Proof Using Remark 1 and Lemma 1, we have $\operatorname{Int} \mathcal{R}(M, \Phi, K) \subset$ Int Dom $\Psi^{*}$.

By part (ii) of Proposition 1, we have

$$
\operatorname{Int} \operatorname{Dom}\left(\Psi^{*}\right)=\left\{g \in \mathbb{R}^{n}:\langle g, w\rangle<\Psi_{\infty}(w), \quad \forall w \in \mathbb{R}^{n} \backslash\{0\}\right\} .
$$

Using (13), we get

$$
\text { Int } \mathcal{R}(M, \Phi, K) \subset\left\{q \in \mathbb{R}^{n}:\langle q, w\rangle+\Phi_{\infty}(w)>0, \quad \forall w \in \operatorname{ker}(M) \cap K_{\infty} \backslash\{0\}\right\} .
$$

Let us prove now the converse, i.e., let $q \in \mathbb{R}^{n}$ such that

$$
\langle q, w\rangle+\Phi_{\infty}(w)>0, \quad \forall w \in \operatorname{ker}(M) \cap K_{\infty} \backslash\{0\} .
$$

We prove that $-q \in \mathcal{R}(M, \Phi, K)$. Let $\left(\varepsilon_{n}\right)_{n}$ be a sequence of non-negative real numbers such that $\varepsilon_{n} \rightarrow 0^{+}$as $n \rightarrow+\infty$. Let $u_{n} \in K$ be the unique solution of problem $\mathrm{VI}\left(M+\varepsilon_{n} I, \Phi, q, K\right)$. We claim that the sequence $\left(u_{n}\right)$ is bounded. Arguing by contradiction, suppose that there exists a subsequence, still denoted $\left(u_{n}\right)$ such that $\left\|u_{n}\right\| \rightarrow+\infty$ as $n \rightarrow+\infty$. We set $w_{n}=\frac{u_{n}}{\left\|u_{n}\right\|}$ and along a subsequence, we may suppose that $w_{n} \rightarrow w \neq 0$. It is clear that $w \in K_{\infty}$. We have

$$
\left\langle M u_{n}+q, v-u_{n}\right\rangle+\varepsilon_{n}\left\langle u_{n}, v-u_{n}\right\rangle+\Phi(v)-\Phi\left(u_{n}\right) \geq 0, \quad \forall v \in K .
$$

Setting $v=0$ in (16), we get

$$
\left\langle M u_{n}+q, u_{n}\right\rangle+\varepsilon_{n}\left\|u_{n}\right\|^{2}+\Phi\left(u_{n}\right)-\Phi(0) \leq 0 .
$$

Dividing by $\left\|u_{n}\right\|^{2}$ and passing to the limit as $n \rightarrow+\infty$, we get $\langle M w, w\rangle \leq 0$. Since $M$ is symmetric and positive semidefinite, we have $w \in \operatorname{ker}(M)$ and consequently $w \in$ $\operatorname{ker}(M) \cap K_{\infty} \backslash\{0\}$.

On the other hand dividing (16) by $t_{n}=\left\|u_{n}\right\|$ and using the fact that $\langle M w, w\rangle \geq 0$, we obtain

$$
\left\langle q, w_{n}\right\rangle+\frac{\Phi\left(t_{n} w_{n}\right)}{t_{n}}-\frac{\Phi(0)}{t_{n}} \leq 0 .
$$

Passing to the limit, we get

$$
\langle q, w\rangle+\Phi_{\infty}(w) \leq 0,
$$

which is a contradiction to the condition (15). Thus the sequence $\left(u_{n}\right)$ is bounded and there exists a subsequence, again denoted $\left(u_{n}\right)$ such that $u_{n} \rightarrow u$ as $n \rightarrow+\infty$. Passing to the limit as $n \rightarrow+\infty$ in (16), we show that $u$ is a solution of $\operatorname{VI}(M, q, \Phi, K)$. Hence $-q \in \mathcal{R}(M, \Phi, K)$. Therefore,

$$
\text { Int } \operatorname{Dom}\left(\Psi^{*}\right) \subset \mathcal{R}(M, \Phi, K),
$$


which implies that

$$
\text { Int } \operatorname{Dom}\left(\Psi^{*}\right) \subset \operatorname{Int} \mathcal{R}(M, \Phi, K) \text {. }
$$

Consequently,

$\operatorname{Int} \mathcal{R}(M, \Phi, K)=\operatorname{Int} \operatorname{Dom}\left(\Psi^{*}\right)=\left\{q \in \mathbb{R}^{n}:\langle q, w\rangle+\Phi_{\infty}(w)>0, \quad \forall w \in \operatorname{ker}(M) \cap\right.$ $\left.K_{\infty} \backslash\{0\}\right\}$,

which completes the proof of Proposition 4.

The following result characterizes the non-emptyness of the topological interior of the resolvent set $\mathcal{R}(M, \Phi, K)$ associated to the variational inequality $\operatorname{VI}(M, q, \Phi, K)$.

Proposition 5 Suppose that assumptions $(\mathcal{H})$ hold. We have

$$
\begin{aligned}
\operatorname{Int} \mathcal{R}(M, \Phi, K) & \neq \emptyset \Longleftrightarrow-\left(\operatorname{ker}(M) \cap K_{\infty} \cap \operatorname{ker}\left(\Phi_{\infty}\right)\right) \bigcap\left(\operatorname{ker}(M) \cap K_{\infty} \cap \operatorname{ker}\left(\Phi_{\infty}\right)\right) \\
& =\{0\},
\end{aligned}
$$

i.e., the cone $\left(\operatorname{ker}(M) \cap K_{\infty} \cap \operatorname{ker}\left(\Phi_{\infty}\right)\right)$ is pointed.

Proof We have

$$
\operatorname{Int} \mathcal{R}(M, \Phi, K) \neq \varnothing \Longleftrightarrow \operatorname{Int} \operatorname{Dom}\left(\Psi^{*}\right) \neq \varnothing .
$$

On the other hand, we have

$$
\left(\operatorname{Dom}\left(\Psi^{*}\right)\right) \times\{-1\}=\mathcal{B}(\text { epi } \Psi) \cap\left(\mathbb{R}^{n} \times\{-1\}\right),
$$

where $\mathcal{B}($ epi $\Psi)$ is the barrier cone to epi $(\Psi)$.

Indeed, let $p \in \operatorname{Dom} \Psi^{*}$ be given. Then there exists $M_{p} \in \mathbb{R}$ such that

$$
\langle p, x\rangle-\Psi(x) \leq M_{p}, \quad \forall x \in \mathbb{R}^{n},
$$

which is equivalent to

$$
\langle(p,-1),(x, \Psi(x))\rangle_{\mathbb{R}^{n} \times \mathbb{R}} \leq M_{p}, \quad \forall x \in \mathbb{R}^{n} .
$$

Therefore,

$$
\langle(p,-1),(x, \lambda)\rangle_{\mathbb{R}^{n} \times \mathbb{R}} \leq M_{p}, \quad \forall(x, \lambda) \in \operatorname{epi}(\Psi) .
$$

Hence,

$$
(p,-1) \in \mathcal{B}(\text { epi } \Psi) .
$$

Now let $(p,-1) \in \mathcal{B}($ epi $\Psi) \cap\left(\mathbb{R}^{n} \times\{-1\}\right)$. Then there existe $M_{p} \in \mathbb{R}^{n}$ such that

$$
\langle(p,-1),(x, \lambda)\rangle_{\mathbb{R}^{n} \times \mathbb{R}} \leq M_{p}, \quad \forall(x, \lambda) \in \operatorname{epi}(\Psi) .
$$

In particular for $(x, \lambda)=(x, \Psi(x))$, we have

$$
\langle p, x\rangle-\Psi(x) \leq M_{p}, \quad \forall x \in \mathbb{R}^{n} .
$$

Consequently,

$$
(p,-1) \in\left(\operatorname{Dom}\left(\Psi^{*}\right)\right) \times\{-1\} .
$$


Using (17) and (18), we have

$$
\text { Int } \mathcal{R}(M, \Phi, K) \neq \emptyset \Longleftrightarrow \operatorname{Int} \mathcal{B}(\text { epi } \Psi) \neq \emptyset \text {. }
$$

Using Proposition 3 for $C=$ epi $\Psi$, we have

$$
\text { Int } \mathcal{B}(\text { epi } \Psi) \neq \emptyset \Longleftrightarrow\left((\text { epi } \Psi)_{\infty}\right) \cap\left(-(\text { epi } \Psi)_{\infty}\right)=\{0\} .
$$

Since, $(\text { epi } \Psi)_{\infty}=$ epi $\Psi_{\infty}$ we get

$$
\text { Int } \mathcal{R}(M, \Phi, K) \neq \varnothing \Longleftrightarrow\left(\left(\text { epi } \Psi_{\infty}\right)\right) \cap\left(-\left(\text { epi } \Psi_{\infty}\right)\right)=\{0\} .
$$

Since $\Phi$ is bounded from below, then $\Psi$ is also bounded from below. Hence, $\Psi_{\infty} \geq 0$ and it is then easy to check that:

$$
\left(\left(\operatorname{epi} \Psi_{\infty}\right)\right) \cap\left(-\left(\operatorname{epi} \Psi_{\infty}\right)\right)=\left(\left(\operatorname{ker} \Psi_{\infty}\right) \times\{0\}\right) \cap\left(-\left(\operatorname{ker} \Psi_{\infty}\right) \times\{0\}\right) .
$$

Therefore,

$$
\text { Int } \mathcal{R}(M, \Phi, K) \neq \emptyset \Longleftrightarrow\left(\left(\operatorname{ker} \Psi_{\infty}\right) \times\{0\}\right) \cap\left(-\left(\operatorname{ker} \Psi_{\infty}\right) \times\{0\}\right)=\{0\} .
$$

Consequently,

$$
\operatorname{Int} \mathcal{R}(M, \Phi, K) \neq \emptyset \Longleftrightarrow\left(\left(\operatorname{ker} \Psi_{\infty}\right)\right) \cap\left(-\left(\operatorname{ker} \Psi_{\infty}\right)\right)=\{0\} .
$$

Using (13), it is easy to see that

$$
\begin{aligned}
\operatorname{Int} \mathcal{R}(M, \Phi, K) & \neq \emptyset \Longleftrightarrow-\left(\operatorname{ker}(M) \cap K_{\infty} \cap \operatorname{ker}\left(\Phi_{\infty}\right)\right) \bigcap\left(\operatorname{ker}(M) \cap K_{\infty} \cap \operatorname{ker}\left(\Phi_{\infty}\right)\right) \\
& =\{0\},
\end{aligned}
$$

which completes the proof of Proposition 5.

\section{A stability result}

Let us now study the stability of the variational inequality $\operatorname{VI}(M, q, \Phi, K)$ in the following sense.

Definition 2 One says that the variational inequality $\operatorname{VI}(M, q, \Phi, K)$ is stable provided that there exists $\varepsilon>0$ such that for any symmetric and positive semidefinite matrix $M_{\varepsilon}$, any vector $q_{\varepsilon} \in q+\varepsilon \mathbb{B}_{n}$ (here $\mathbb{B}_{n}$ denotes the open unit ball in $\mathbb{R}^{n}$ ), any proper lower semicontinuous bounded from below convex function $\Phi_{\varepsilon}$, and any non-empty closed convex set $K_{\varepsilon}$ satisfying the following conditions

$$
0 \in \operatorname{Dom} \Phi_{\varepsilon} \cap K_{\varepsilon} \text { and } \operatorname{ker}(M) \cap \operatorname{ker}\left(\Phi_{\infty}\right) \cap K_{\infty}=\operatorname{ker}\left(M_{\varepsilon}\right) \cap \operatorname{ker}\left(\left(\Phi_{\varepsilon}\right)_{\infty}\right) \cap\left(K_{\varepsilon}\right)_{\infty},
$$

the perturbed problem $\operatorname{VI}\left(M_{\varepsilon}, q_{\varepsilon}, \Phi_{\varepsilon}, K_{\varepsilon}\right)$ has at least one solution.

Before starting our study, let us first give some simple examples to motivate the notion of stability of $\operatorname{VI}(M, q, \Phi, K)$ with respect to small perturbations.

Example 1 Set

$$
M=\left(\begin{array}{ll}
0 & 0 \\
0 & 0
\end{array}\right), \quad K_{1}=\left\{(x, y) \in \mathbb{R}^{2}: y \geq 0\right\}, \quad \Phi \equiv 0 .
$$


In this case the resolvent set is given by

$$
\mathcal{R}\left(M, 0, K_{1}\right)=\left\{\left(q_{1}, q_{2}\right) \in \mathbb{R}^{2}: q_{1}=0 \text { and } q_{2} \leq 0\right\} .
$$

We note that in this case $\operatorname{Int} \mathcal{R}\left(M, 0, K_{1}\right)=\emptyset$. Hence problem $\operatorname{VI}\left(M, q, 0, K_{1}\right)$ is not stable with respect to small perturbations of the right hand term $q$. More precisely, if we replace $q$ by $q_{\varepsilon}$ such that $\left\|q-q_{\varepsilon}\right\| \leq \varepsilon$ for a given $\varepsilon>0$, the solution $\operatorname{set} \operatorname{Sol}\left(M, q_{\varepsilon}, 0, K_{1}\right)$ of the perturbed problem may be empty.

We note that in this case, we have

$$
\operatorname{ker}(M) \cap K_{1 \infty} \cap \operatorname{ker}\left(\Phi_{\infty}\right)=\mathbb{R} \times \mathbb{R}^{+},
$$

which is not a pointed cone.

Example 2 Let $M$ and $\Phi$ as in Example 1 Consider now, the convex and closed subset $K_{2}$ given by

$$
K_{2}=\left\{(x, y) \in \mathbb{R}^{2}: y \geq x^{2}\right\} .
$$

In this case the resolvent set is given by

$$
\mathcal{R}\left(M, 0, K_{2}\right)=\left\{\left(q_{1}, q_{2}\right) \in \mathbb{R}^{2}: q_{2}<0\right\} \cup\{(0,0)\} .
$$

Here Int $\mathcal{R}\left(M, 0, K_{2}\right) \neq \varnothing$ and problem $V I\left(M, q, 0, K_{2}\right)$ is stable with respect to small perturbations of the right hand term $q$.

We note that in this case, we have

$$
\operatorname{ker}(M) \cap K_{2 \infty} \cap \operatorname{ker}\left(\Phi_{\infty}\right)=\{0\} \times \mathbb{R}^{+},
$$

which is a pointed cone.

We have the following existence and stability result related to the linear variational inequality $\operatorname{VI}(M, q, \Phi, K)$.

Theorem 1 Assume that assumptions $(\mathcal{H})$ are satisfied. Then the variational inequality $\operatorname{VI}(M, q, \Phi, K)$ is stable in the sense of Definition 2 if and only if

$$
\langle q, w\rangle+\Phi_{\infty}(w)>0, \quad \forall w \in \operatorname{ker}(M) \cap K_{\infty}, \quad w \neq 0 .
$$

Proof We know from Proposition 4 that if

$$
\langle q, w\rangle+\Phi_{\infty}(w)>0, \quad \forall w \in \operatorname{ker}(M) \cap K_{\infty} \backslash\{0\}
$$

then

$$
-q \in \operatorname{Int} \mathcal{R}(M, \Phi, K) \neq \emptyset .
$$

Therefore, there exists $\varepsilon>0$ such that

$$
-q+\varepsilon \mathbb{B}_{n} \subset \operatorname{Int} \mathcal{R}(M, \Phi, K) .
$$

Let now $M_{\varepsilon}$ be a symmetric and positive semidefinite matrix, $q_{\varepsilon} \in q+\varepsilon \mathbb{B}_{n}$, $\Phi_{\varepsilon}$ be a proper lower semicontinuous bounded from below convex function and $K_{\varepsilon}$ be a non-empty closed convex set satisfying

$$
0 \in \operatorname{Dom} \Phi_{\varepsilon} \cap K_{\varepsilon}
$$


and

$$
\operatorname{ker}(M) \cap \operatorname{ker}\left(\Phi_{\infty}\right) \cap K_{\infty}=\operatorname{ker}\left(M_{\varepsilon}\right) \cap \operatorname{ker}\left(\left(\Phi_{\varepsilon}\right)_{\infty}\right) \cap\left(K_{\varepsilon}\right)_{\infty}
$$

Using Proposition 4, we have

$$
\operatorname{Int} \mathcal{R}(M, \Phi, K)=\operatorname{Int} \mathcal{R}\left(M_{\varepsilon}, \Phi_{\varepsilon}, K_{\varepsilon}\right) .
$$

Therefore,

$$
-q_{\varepsilon} \in-q+\varepsilon \mathbb{B} \subset \operatorname{Int} \mathcal{R}\left(M_{\varepsilon}, \Phi_{\varepsilon}, K_{\varepsilon}\right) .
$$

Consequently, $\operatorname{Sol}\left(M_{\varepsilon}, q_{\varepsilon}, \Phi_{\varepsilon}, K_{\varepsilon}\right) \neq \varnothing$. This ensures that the variational inequality $\operatorname{VI}(M, q, \Phi, K)$ is stable in the sense of Definition 2.

Suppose now that the variational inequality $\operatorname{VI}(M, q, \Phi, K)$ is stable in the sense of Definition 2. Then there exists $\varepsilon>0$ such that (taking $M_{\varepsilon}=M, \Phi_{\varepsilon}=\Phi$ and $K_{\varepsilon}=K$ ) for every $q_{\varepsilon} \in q+\varepsilon \mathbb{B}_{n}, \operatorname{Sol}\left(M, q_{\varepsilon}, \Phi, K\right) \neq \emptyset$. Therefore,

$$
-q \in \operatorname{Int} \mathcal{R}(M, \Phi, K) .
$$

Then using Proposition 4, we obtain

$$
\langle q, w\rangle+\Phi_{\infty}(w)>0, \quad \forall w \in \operatorname{ker}(M) \cap K_{\infty}, \quad w \neq 0,
$$

which completes the proof of Theorem 1 .

Remark 3 It is clear that any symmetric and positive semidefinite matrix $M_{\varepsilon}$ such that $\operatorname{ker}(M)=\operatorname{ker}\left(M_{\varepsilon}\right)$ and any function $\Phi_{\varepsilon} \in \Gamma_{0}\left(\mathbb{R}^{n}\right)$ bounded from below such that $\Phi_{\infty}=$ $\left(\Phi_{\varepsilon}\right)_{\infty}$ and any non-empty closed convex set $K_{\varepsilon}$ such that $K_{\infty}=\left(K_{\varepsilon}\right)_{\infty}$ satisfy condition (25). This the case for example if we take $M=M_{\varepsilon}, \Phi-\varepsilon \leq \Phi_{\varepsilon} \leq \Phi+\varepsilon$, $K \subset K_{\varepsilon}+\varepsilon \mathbb{B}_{n}$ and $K_{\varepsilon} \subset K+\varepsilon \mathbb{B}_{n}$.

We have the following consequence of Theorem 1.

Corollary 1 Assume that assumptions $(\mathcal{H})$ are satisfied. If the following compatibility condition

$$
\langle q, w\rangle+\Phi_{\infty}(w)>0, \quad \forall w \in \operatorname{ker}(M) \cap K_{\infty}, \quad w \neq 0,
$$

is satisfied, then $\operatorname{VI}(M, q, \Phi, K)$ has at least one solution.

Proof Take $M_{\varepsilon}=M, \Phi_{\varepsilon}=\Phi, q_{\varepsilon}=q$ and $K_{\varepsilon}=K$ in Theorem 1 .

\section{Some applications}

We give in this section some applications of Theorem 1 and Corollary 1.

Example 3 (Clipping circuit 1/Ideal diode) Let us consider the circuit of Fig. 1 involving a load resistance $R>0$, an input-signal source $u$ and corresponding instantaneous current $i$, an ideal diode as a shunt element and a supply voltage $E$.

Figure 2 illustrates the ampere-volt characteristic of an ideal diode.

This is a model in which the diode is a simple switch. If $V<0$ then $i=0$ and the diode is blocking. If $i>0$ then $V=0$ and the diode is conducting. We first see that the ideal diode is described by the complementarity relation

$$
V \leq 0, \quad i \geq 0, \quad V i=0
$$


Fig. 1 Clipping circuit 1: diode as shunt element
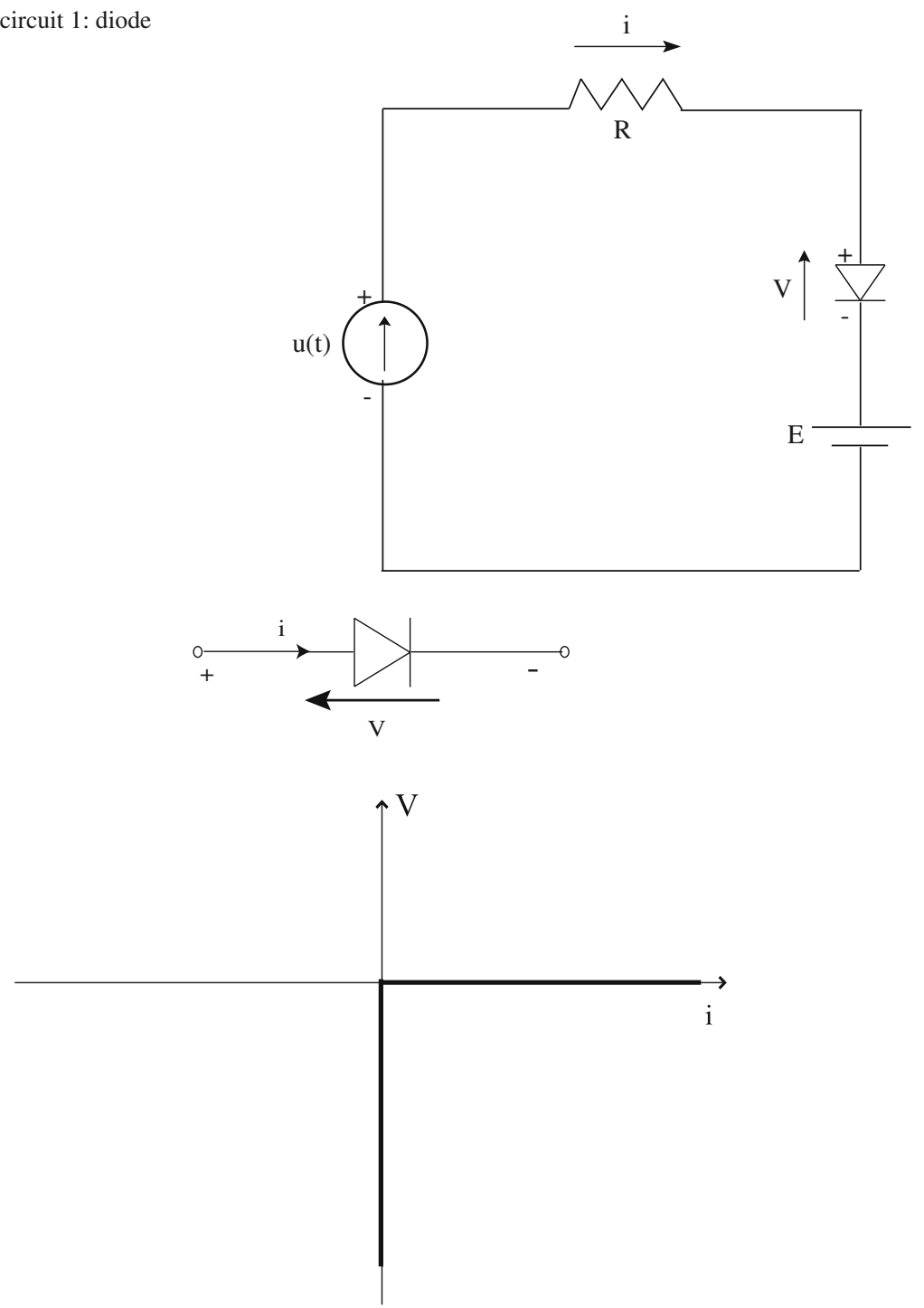

Fig. 2 Ideal diode model

that is also

$$
\min \{-V, i\}=0 .
$$

The electrical superpotential of the ideal diode is

$$
\varphi_{D}(x)=I_{\mathbb{R}_{+}}(x), \quad(x \in \mathbb{R})
$$

Then

$$
\varphi_{D}^{*}(z)=I_{\mathbb{R}_{-}}(z), \quad(z \in \mathbb{R})
$$


and the recession function of the electrical superpotential is:

$$
\left(\varphi_{D}\right)_{\infty}(x)=\varphi_{D}(x),(x \in \mathbb{R}) .
$$

We have also

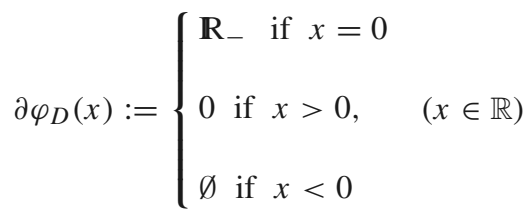

and

$$
\partial \varphi_{D}^{*}(z):=\left\{\begin{array}{l}
\mathbf{R}_{+} \text {if } z=0 \\
0 \text { if } z<0, \quad(z \in \mathbb{R}) . \\
\emptyset \text { if } z>0
\end{array}\right.
$$

The complementarity relation can be written as

$$
V \in \partial \varphi_{D}(i) \Longleftrightarrow i \in \partial \varphi_{D}^{*}(V) \Longleftrightarrow \varphi_{D}(i)+\varphi_{D}^{*}(V)=i V .
$$

Kirchoff's voltage law gives

$$
u=U_{R}+V_{D}+E
$$

where $U_{R}=R i$ denotes the difference of potential across the resistor and $V_{D} \in \partial I_{\mathbf{R}_{+}}(i)$ is the difference of potential across diode. Thus

$$
E+R i-u \in-\partial I_{\mathbf{R}_{+}}(i)
$$

which is equivalent to $\mathbf{V I}\left(\mathbf{R}, \mathbf{E}-\mathbf{u}, \mathbf{0}, \mathbf{R}_{+}\right)$, i.e.,

$$
i \in \mathbb{R}_{+}:(R i+E-u)(v-i) \geq 0, \quad \forall v \in \mathbb{R}_{+} .
$$

Here $R>0$ and for each $E, u \in \mathbb{R}$, we may apply Theorem 1 to assert that (30) is stable in the sense of Definition 2.

Moreover:

$$
\begin{aligned}
(30) & \Longleftrightarrow \frac{E}{R}+i-\frac{u}{R} \in-\partial I_{\mathbb{R}_{+}}(i) \Longleftrightarrow-\frac{E}{R}+\frac{u}{R} \in i+\partial I_{\mathbb{R}_{+}}(i) \\
& \Longleftrightarrow i=\left(i d_{\mathbb{R}}+\partial I_{\mathbb{R}_{+}}\right)^{-1}\left(\frac{u-E}{R}\right)=\frac{1}{R} \max \{0, u-E\} .
\end{aligned}
$$

If $u \leq E$ then the diode is blocking while if $u>E$ then the diode is conducting.

Let us now consider a driven time depending input $t \mapsto u(t)$ and define the output-signal $t \mapsto V_{o}(t)$ as

$$
V_{o}(t)=E+V(t) .
$$

The time depending current $t \mapsto i(t)$ is given by

$$
i(t)=\frac{1}{R} \max \{0, u(t)-E\}
$$

and thus

$$
V_{o}(t)=V(t)+E=u(t)-R i(t)=u(t)+\min \{0, E-u(t)\}=\min \{u(t), E\} .
$$


Fig. 3 Practical diode model

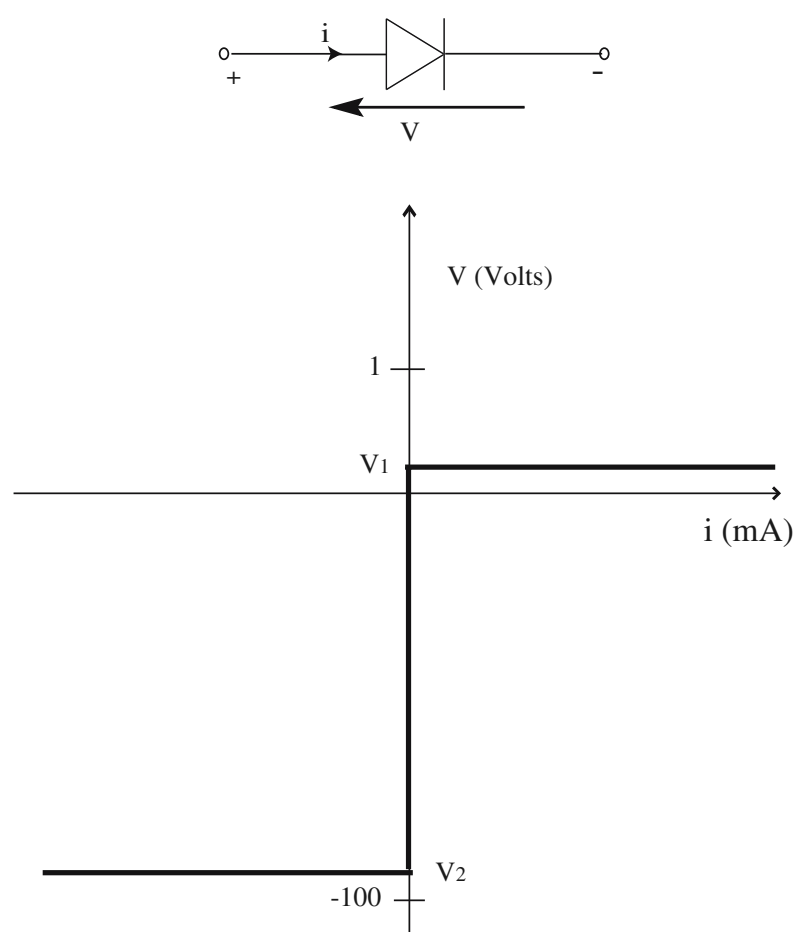

This shows that the circuit in Fig. 1 can be used to transmit the part of a given input-signal $u$ which lies below some given reference level $E$.

Example 4 (Clipping circuit 1/concrete diode) Let us now consider the circuit in Fig. 1 with a concrete diode.

Figure 3 illustrates the ampere-volt characteristic of a practical diode model.

There is a voltage point, called the knee voltage $V_{1}$, at which the diode begins to conduct and a maximum reverse voltage, called the peak reverse voltage $V_{2}$, that will not force the diode to conduct. When this voltage is exceeded, the depletion may breakdown and allow the diode to conduct in the reverse direction. Note that usually $\left|V_{1}\right| \ll\left|V_{2}\right|$ and the model is locally ideal.

For general purpose diodes used in low frequency/speed applications, $\left|V_{1}\right| \simeq 0.7-2.5 \mathrm{~V}$ and $\left|V_{2}\right| \simeq 5 \mathrm{kV}$; for high voltage rectifier diodes, $\left|V_{1}\right| \simeq 10 \mathrm{~V}$ and $\left|V_{2}\right| \simeq 30 \mathrm{kV}$; for fast diodes used in switched mode power supply and inverter circuits, $\left|V_{1}\right| \simeq 0.7-1.5 \mathrm{~V}$ and $\left|V_{2}\right| \simeq 3 \mathrm{kV}$ and for Schottky diodes used in high frequency applications, $\left|V_{1}\right|$ $\simeq 0.2-0.9 \mathrm{~V}$ and $\left|V_{2}\right| \simeq 100 \mathrm{~V}$.

The electrical superpotential of the practical diode is

$$
\varphi_{P D}(x)=\left\{\begin{array}{l}
V_{1} x \text { if } x \geq 0 \\
V_{2} x \text { if } x<0
\end{array}, \quad(x \in \mathbb{R}) .\right.
$$

Then

$$
\varphi_{P D}^{*}(z)=I_{\left[V_{2}, V_{1}\right]}(z), \quad(z \in \mathbb{R})
$$



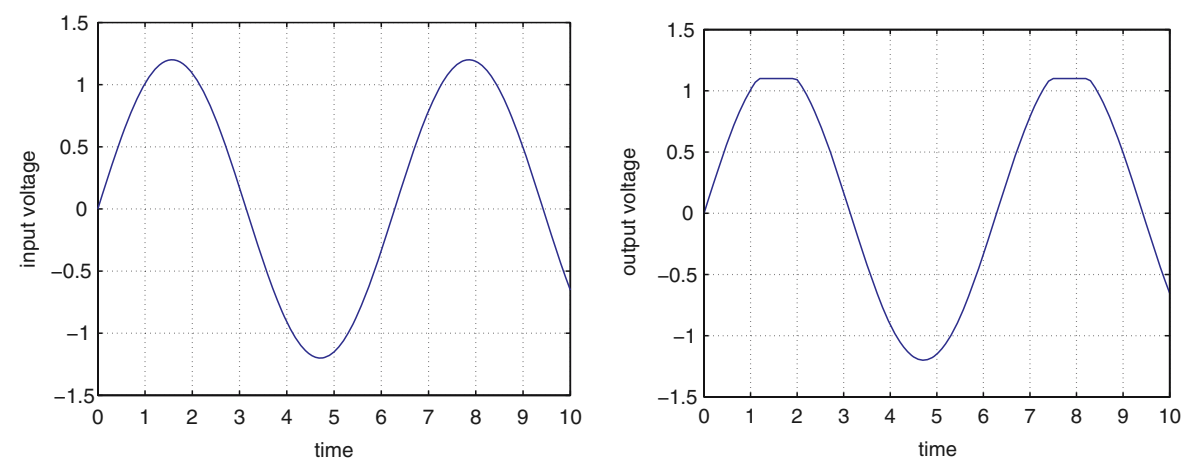

Fig. 4 Clipping circuit 1: general diode as shunt element using, $V_{1}=0.1, V_{2}=-90, E=1$

and the recession function of the electrical superpotential is given by:

$$
\left(\varphi_{P D}\right)_{\infty}(x)=\varphi_{P D}(x), \quad(x \in \mathbb{R}) .
$$

We see that

$$
\partial \varphi_{P D}(x)=\left\{\begin{array}{l}
V_{2} \text { if } x<0 \\
{\left[V_{2}, V_{1}\right] \text { if } x=0, \quad(x \in \mathbb{R})} \\
V_{1} \text { if } x>0
\end{array}\right.
$$

recovers the ampere-volt characteristic $(i, V)$ while

$$
\partial \varphi_{P D}^{*}(z)=\left\{\begin{array}{l}
\mathbb{R}_{-} \text {if } z=V_{2} \\
0 \text { if } z \in] V_{2}, V_{1}[ \\
\mathbb{R}_{+} \text {if } z=V_{1} \\
\varnothing \text { if } z \in \mathbb{R} \backslash\left[V_{2}, V_{1}\right]
\end{array}, \quad(z \in \mathbb{R}) .\right.
$$

recovers the volt-ampere characteristic $(V, i)$. The ampere-volt characteristic of the practical diode (Fig. 4) can thus be written as

$$
V \in \partial \varphi_{P D}(i) \Longleftrightarrow i \in \partial \varphi_{P D}^{*}(V) \Longleftrightarrow \varphi_{P D}(i)+\varphi_{P D}^{*}(V)=i V .
$$

We may follow the same steps as in the previous example to see that Kirchoff's law reduces to $\mathbf{V I}\left(\mathbf{R}, \mathbf{E}-\mathbf{u}, \varphi_{\mathbf{P D}}, \mathbb{R}\right)$, i.e.,

$$
i \in K:=\mathbb{R}:(R i+E-u)(v-i)+\varphi_{P D}(v)-\varphi_{P D}(i) \geq 0, \quad \forall v \in \mathbb{R} .
$$

Here $R>0$ and for each $E, u \in \mathbb{R}$, we may apply Theorem 1 to assert that (4) is stable in the sense of Definition 2. Moreover:

$$
i(t)=\left(i d_{\mathbb{R}}+\partial \varphi_{P D}\right)^{-1}\left(\frac{u(t)-E}{R}\right)=\operatorname{argmin}_{x \in \mathbb{R}}\left\{\frac{1}{2}\left|x-\left(\frac{u(t)-E}{R}\right)\right|^{2}+\varphi_{P D}(x)\right\} .
$$

and

$$
V_{o}(t)=u(t)-R i(t)
$$


Fig. 5 Double-diode clipper

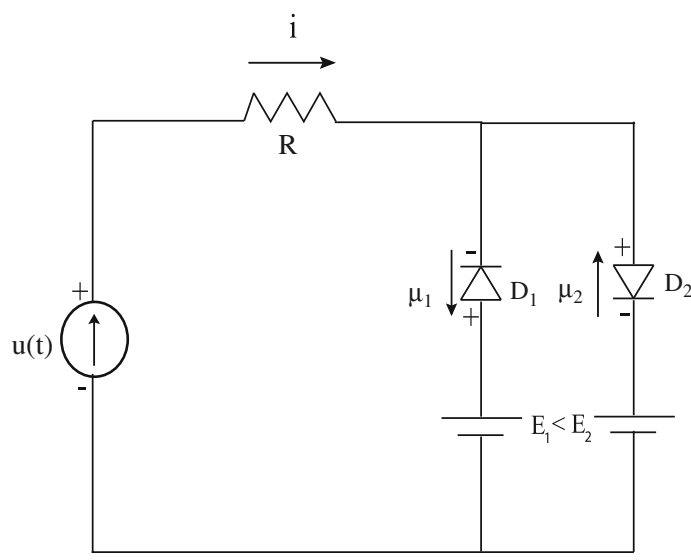

Example 5 Let us consider the double-diode clipper circuit in Fig. 5 involving a load resistance $R>0$, two ideal diodes, an input-signal source and two supply voltages $E_{1}$ and $E_{2}$. It is assumed that $E_{1}<E_{2}$. We denote by $i$ the current through the resistor $R$ and we set $i=i_{1}+i_{2}$ where $-i_{1}$ denotes the current through diode $D_{1}$ and $i_{2}$ is the current through diode $D_{2}$. We denote by $\mu_{1}$ the difference of potential across diode $D_{1}$ and by $\mu_{2}$ the difference of potential across diode $D_{2}$.

Using Kirchoff's voltage laws, we get the system:

$$
\left\{\begin{array}{l}
E_{1}+R\left(i_{1}+i_{2}\right)-u=+\mu_{1} \\
E_{2}+R\left(i_{1}+i_{2}\right)-u=-\mu_{2}
\end{array}\right.
$$

The ideal diodes $D_{1}$ and $D_{2}$ are simple switch. If $\mu_{1}<0$ (resp. $\mu_{2}<0$ ) then $-i_{1}=0$ (resp. $\left.i_{2}=0\right)$ and the diode is blocking while if $-i_{1}>0$ (resp. $\left.i_{2}>0\right)$ then $\mu_{1}=0$ (resp. $\left.\mu_{2}=0\right)$ and the diode is conducting. Thus

$$
\mu_{1} \leq 0, \quad-i_{1} \geq 0, \quad \mu_{1} i_{1}=0
$$

and

$$
\mu_{2} \leq 0, \quad-i_{2} \geq 0, \quad \mu_{2} i_{2}=0 .
$$

These last complementarity relations can also be written as:

$$
\mu_{1} \in \partial I_{\mathbb{R}_{+}}\left(-i_{1}\right)=-I_{\mathbb{R}_{-}}\left(i_{1}\right)
$$

and

$$
\mu_{2} \in \partial I_{\mathbb{R}_{+}}\left(i_{2}\right)
$$

Setting

$$
K=\mathbb{R}_{-} \times \mathbb{R}_{+}, \quad M=\left(\begin{array}{c}
R \\
R \\
R
\end{array}\right), \quad q=\left(\begin{array}{c}
E_{1}-u \\
E_{2}-u
\end{array}\right), \quad I=\left(\begin{array}{c}
i_{1} \\
i_{2}
\end{array}\right),
$$

we see that the system in (35) is equivalent to the variational inequality $\operatorname{VI}(\mathbf{M}, \mathbf{q}, \mathbf{0}, \mathbf{K})$, i.e.,

$$
I \in K:\langle M I+q, v-I\rangle \geq 0, \quad \forall v \in K .
$$


Here the matrix $M$ is positive semidefinite and symmetric,

$$
K_{\infty} \equiv K, \operatorname{ker}\{M\}=\left\{v \in \mathbb{R}^{2}: v_{2}=-v_{1}\right\}
$$

and

$$
K_{\infty} \cap \operatorname{ker}\{M\}=\{(-\alpha, \alpha) ; \alpha \geq 0\} .
$$

Moreover, for all $v \in K_{\infty} \cap \operatorname{ker}\{M\}, v \neq(0,0)$ there exists $\alpha>0$ such that $v=(-\alpha, \alpha)$ and

$$
\langle q, v\rangle=\left(E_{1}-u\right) v_{1}+\left(E_{2}-u\right) v_{2}=\alpha\left(E_{2}-E_{1}\right)>0 .
$$

We may thus apply Theorem 1 to assert that a double-diode clipper involving ideal diodes is stable with respect to data perturbations in the sense of Definition 2.

Moreover, using the relations in (35) we see that:

$$
i_{1}^{*}+i_{2}^{*}=\min \left\{i_{2}^{*}, \frac{u-E_{1}}{R}\right\}=\max \left\{i_{1}^{*}, \frac{u-E_{2}}{R}\right\}
$$

from which we deduce, after elementary calculations, that:

$$
i^{*}=\left\{\begin{array}{l}
\frac{u-E_{1}}{R} \text { if } u<E_{1} \\
0 \text { if } E_{1} \leq u \leq E_{2} . \\
\frac{u-E_{2}}{R} \text { if } u>E_{2}
\end{array} .\right.
$$

So, for a driven time depending input $t \mapsto u(t)$ the time depending current $t \mapsto i^{*}(t)$ through the resistor $R$ is given by

$$
i^{*}(t)=\left\{\begin{array}{l}
\frac{u(t)-E_{1}}{R} \text { if } u(t)<E_{1} \\
0 \text { if } E_{1} \leq u(t) \leq E_{2} \\
\frac{u(t)-E_{2}}{R} \text { if } u(t)>E_{2}
\end{array}\right.
$$

and the output-signal $t \mapsto V_{o}(t)$ defined by

$$
V_{o}(t)=V_{2}(t)+E_{2}=u(t)-R i^{*}(t)
$$

is then given by the expression:

$$
V_{o}(t)=\left\{\begin{array}{l}
E_{1} \text { if } u(t)<E_{1} \\
u(t) \text { if } E_{1} \leq u(t) \leq E_{2} . \\
E_{2} \text { if } u(t)>E_{2}
\end{array}\right.
$$

This shows that the circuit can be used to transmit the part of a given input-signal $u$ that lies above some level $E_{1}$ and below some level $E_{2}$ (Fig. 6).

Example 6 Let us again consider the circuit in Fig. 5 and suppose that the electrical superpotential of each diodes $D_{1}$ and $D_{2}$ is given by (practical diode model):

$$
\varphi_{P D}(x)=\left\{\begin{array}{l}
V_{1} x \text { if } x \geq 0 \\
V_{2} x \text { if } x<0
\end{array}, \quad(x \in \mathbb{R})\right.
$$



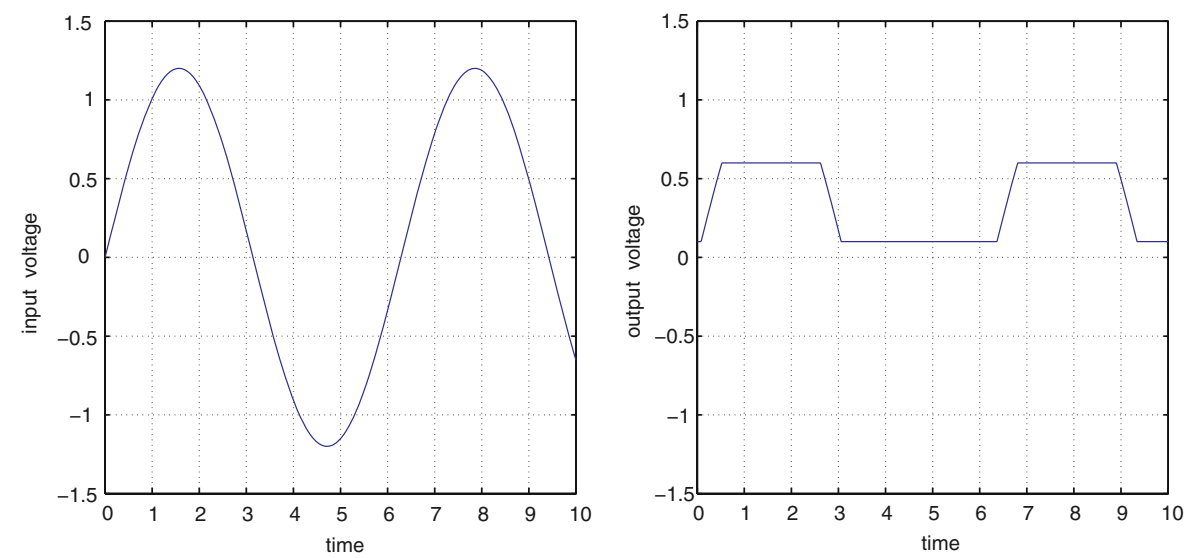

Fig. 6 Double-diode clipper: ideal diode, $E_{1}=0.1, E_{2}=0.6$

where $V_{2}<0<V_{1}$. We suppose also that

$$
\left|V_{2}\right|>\frac{E_{2}-E_{1}}{2} \text {. }
$$

We set

$$
\bar{\varphi}_{P D}(x)=\varphi_{P D}(-x), \quad \forall x \in \mathbb{R}
$$

and

$$
\Phi(x)=\bar{\varphi}_{P D}\left(x_{1}\right)+\varphi_{P D}\left(x_{2}\right), \quad \forall\left(x_{1}, x_{2}\right) \in \mathbb{R}^{2} .
$$

Kirchoff's laws yield the system

$$
\left\{\begin{array}{l}
E_{1}+R\left(i_{1}+i_{2}\right)-u=+\mu_{1} \in-\partial \bar{\varphi}_{P D}\left(i_{1}\right) \\
E_{2}+R\left(i_{1}+i_{2}\right)-u=-\mu_{2} \in-\partial \varphi_{P D}\left(i_{2}\right)
\end{array}\right.
$$

which is equivalent to the variational inequality $\mathbf{V I}\left(\mathbf{M}, \mathbf{q}, \Phi, \mathbf{R}^{\mathbf{2}}\right)$, i.e.,

$$
\Upsilon \in \mathbb{R}^{2}:\langle M \Upsilon+q, v-\Upsilon\rangle+\Phi(v)-\Phi(\Upsilon) \geq 0, \quad \forall v \in \mathbb{R}^{2},
$$

with $M$ and $q$ as in (36) and $\Phi$ as in (42). Here

$$
\operatorname{ker}\{M\}=\left\{v \in \mathbb{R}^{2}: v_{2}=-v_{1}\right\}
$$

Let $v \in \operatorname{ker}\{M\}, v \neq 0$, be given. Then:

$$
\langle q, v\rangle+\Phi_{\infty}(v)=v_{2}\left(E_{2}-E_{1}\right)+\varphi_{P D}\left(-v_{1}\right)+\varphi_{P D}\left(v_{2}\right)=v_{2}\left(E_{2}-E_{1}\right)+2 \varphi_{P D}\left(v_{2}\right) .
$$

It results that if $v_{2}>0$ then

$$
\langle q, v\rangle+\Phi_{\infty}(v)=v_{2}\left(E_{2}-E_{1}\right)+2 V_{1} v_{2}
$$

while if $v_{2}<0$ then

$$
\langle q, v\rangle+\Phi_{\infty}(v)=-v_{2}\left(2\left|V_{2}\right|-\left(E_{2}-E_{1}\right)\right)>0 .
$$

We may then apply Theorem 1 to assert that the variational inequality $\operatorname{VI}\left(\mathbf{M}, \mathbf{q}, \Phi, \mathbb{R}^{\mathbf{2}}\right)$ is stable in the sense of Definition 2. 

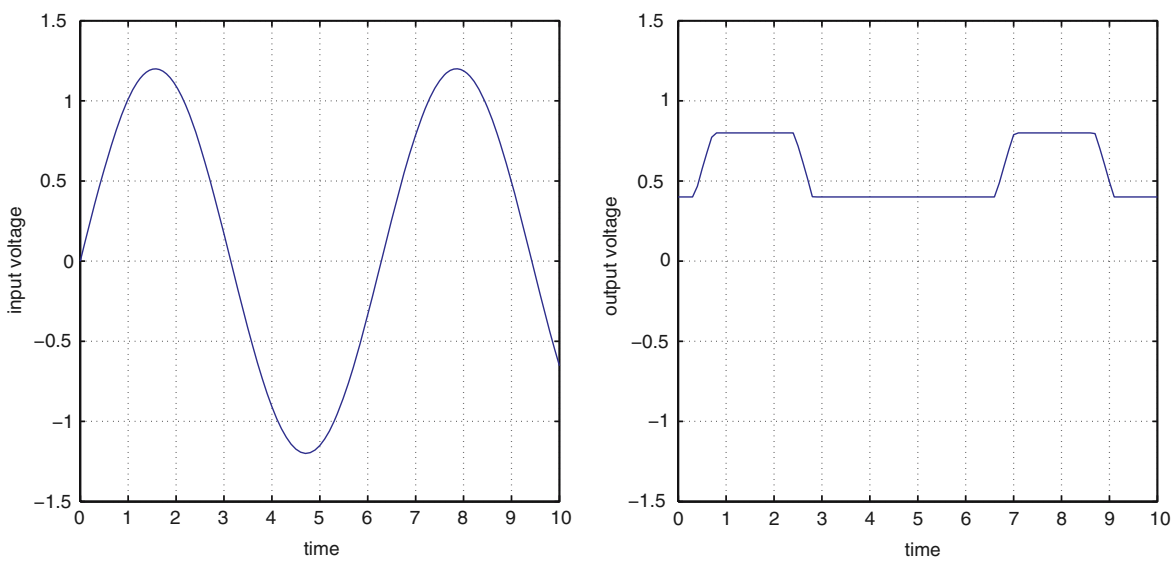

Fig. 7 Double-diode clipper: practical diode

Fig. 8 Two rigid bodies interconnected by a spring

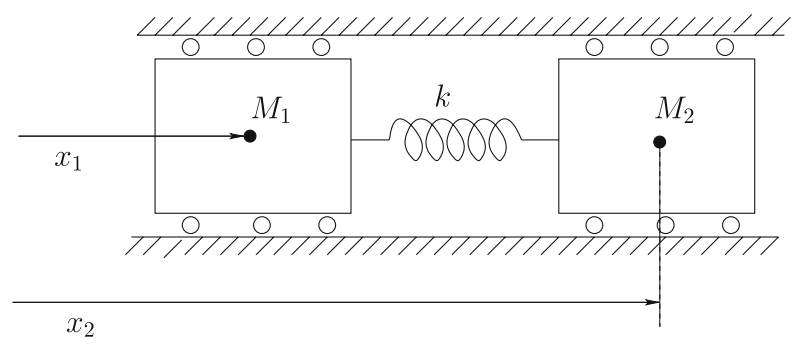

Moreover, the function $\Phi$ is strictly convex and the solution $\Upsilon^{*}$ of (44) is unique and given by:

$$
\Upsilon^{*}=\operatorname{argmin}_{x \in \mathbb{R}^{2}}\left\{\frac{1}{2}\langle M x, x\rangle+\langle q, x\rangle+\Phi(x)\right\} .
$$

So, for a driven time depending input $t \mapsto u(t)$ the time depending current $t \mapsto i^{*}(t)$ through the resistor $R$ (Fig. 7) is given by

$$
i^{*}(t)=i_{1}^{*}(t)+i_{2}^{*}(t)
$$

where

$$
\left(i_{1}^{*}(t) i_{2}^{*}(t)\right)^{T}=\operatorname{argmin}_{x \in \mathbb{R}^{2}}\left\{\frac{1}{2}\langle M x, x\rangle+\left(E_{1}-u(t)\right) x_{1}+\left(E_{2}-u(t)\right) x_{2}+\Phi(x)\right\}
$$

and the output-signal $V_{o}$ can then be determined by the formula:

$$
V_{o}(t)=u(t)-R i^{*}(t) .
$$

Example 7 Let us consider the system of two rigid bodies $M_{1}$ and $M_{2}$ interconnected by a spring of stiffness $k>0$ and constrained to move only in the horizontal direction. The position of $M_{1}$ relative to the origin is represented by $x_{1}$ while the position of $M_{2}$ relative to the origin is determined by $x_{2}$ (Fig. 8).

The mass $M_{1}$ is subjected to some external force $q_{1}$ and static Coulomb friction force $F_{1}$ while the mass $M_{2}$ is subjected to some external force $q_{2}$ and static Coulomb friction $F_{2}$. 
The equilibrium states of the system are characterized by the equilibrium equations:

$$
F_{1}+k\left(x_{2}-x_{1}\right)+q_{1}=0
$$

and

$$
F_{2}-k\left(x_{2}-x_{1}\right)+q_{2}=0 .
$$

Static friction Coulomb models for $F_{1}$ and $F_{2}$ are

$$
F_{1} \in\left\{\begin{array}{l}
-\mu \text { if } x_{1}<0 \\
{[-\mu, \mu] \text { if } x_{1}=0} \\
+\mu \text { if } x_{1}>0
\end{array}\right.
$$

and

$$
F_{2} \in\left\{\begin{array}{l}
-\mu \text { if } x_{2}<0 \\
{[-\mu, \mu] \text { if } x_{2}=0} \\
+\mu \text { if } x_{2}>0
\end{array}\right.
$$

where $\mu>0$ denotes Coulomb friction coefficient. These set-valued relations can also be written as

$$
F_{1} \in-\partial \Psi\left(x_{1}\right)
$$

and

$$
F_{2} \in-\partial \Psi\left(x_{2}\right)
$$

where

$$
\Psi(x)=\mu|x|, \quad(x \in \mathbb{R}) .
$$

Setting

$$
\Phi(x)=\Psi\left(x_{1}\right)+\Psi\left(x_{2}\right), \quad \forall x=\left(x_{1}, x_{2}\right) \in \mathbb{R}^{2}
$$

and

$$
M=\left(\begin{array}{l}
k-k \\
k-k
\end{array}\right), \quad q=\left(\begin{array}{l}
q_{1} \\
q_{2}
\end{array}\right), \quad X=\left(\begin{array}{l}
x_{1} \\
x_{2}
\end{array}\right),
$$

we see that our equilibrium system is equivalent to the variational inequality $\operatorname{VI}(\mathbf{M},-\mathbf{q}$, $\Phi, \mathbb{R}^{2}$ ), i.e.,

$$
X \in \mathbb{R}^{2}:\langle M X+q, v-X\rangle+\Phi(v)-\Phi(X) \geq 0, \quad \forall v \in \mathbb{R}^{2} .
$$

Here the matrix $M$ is positive semidefinite and symmetric. Moreover

$$
\operatorname{ker}\{M\}=\left\{v \in \mathbb{R}^{2}: v_{2}=v_{1}\right\} .
$$

Suppose now that

$$
\left|q_{1}+q_{2}\right|<2 \mu \text {. }
$$


For all $v \in \operatorname{ker}\{M\}, v \neq(0,0)$ there exists $\alpha \neq 0$ such that $v=(\alpha, \alpha)$ and

$$
\Phi_{\infty}(v)+\langle q, v\rangle=2 \mu|\alpha|+\left(q_{1}+q_{2}\right) \alpha .
$$

If $\alpha>0$ then

$$
2 \mu|\alpha|+\left(q_{1}+q_{2}\right) \alpha=\left(2 \mu+\left(q_{1}+q_{2}\right)\right) \alpha>0 .
$$

If $\alpha<0$ then

$$
2 \mu|\alpha|+\left(q_{1}+q_{2}\right) \alpha=\left(2 \mu-\left(q_{1}+q_{2}\right)\right)|\alpha|>0 .
$$

We may thus apply Theorem 1 to assert that our system is stable with respect to data perturbations in the sense of Definition 2.

\section{References}

1. Adly, S., Goeleven, D., Théra, M.: Recession mappings and noncoercive variational inequalities. Nonlinear Anal. 26(9), 1573-1603 (1996)

2. Adly, S., Ernst, E., Théra, M.: Stability of non-coercive variational inequalities. Commun. Contemp. Math. 4(1), 145-160 (2002)

3. Adly, S., Ernst, E., Théra, M.: Well-positioned closed convex sets and well-positioned closed convex functions. J. Glob. Optim. 29, 337-351 (2004)

4. Ang, D.D., Schmidt, K., Vy, K.: Noncoercive variational inequalities: some applications. Nonlinear Anal. TMA 15(6), 497-512 (1990)

5. Baiocchi, C., Gastaldi, F., Tomarelli, F.: Some existence results on noncoercive variational inequalities. Ann. Scuola Norm. Sup. Pisa 13(4), 617-659 (1986)

6. Baiocchi, C., Buttazzo, G., Gastaldi, F., Tomarelli, F.: General existence theorems for unilateral problems in continum mechanics. Arch. Rat. Mech. Anal. 100(2), 149-180 (1988)

7. Brezis, H.: Equations et inéquations non- linéaires dans les espaces vectoriels en dualité. Ann. Inst. Fourier 18(1), 115-175 (1968)

8. Brezis, H.: Problèmes unilatéraux. J. Math. Pures Appl. 51, 1-168 (1972)

9. Browder, F.: Nonlinear maximal monotone mappings in Banach spaces. Math. Ann. 175, 81-113 (1968)

10. Duvaut, G., Lions, J.L.: Les inéquations en mécanique et en physique. Dunod (1972)

11. Fichera G.: Problemi elastostatici con vincoli unilaterali: il problema di Signorini con ambigue condizioni al contorno. Atti. Accad. Naz. Lincei Mem. Sez. I 7(8), 71-140 (1964)

12. Fichera, G.: Boundary value problems in elasticity with unilateral constraints. In: Handbuch der Physik, vol. VIa. 2, pp. 347-389. Springer-Verlag, Berlin Heidelberg New York (1972)

13. Goeleven, D., Motreanu, D.: Variational and Hemivariational Inequalities - Theory, Methods and Applications. Vol.2: Unilateral Problems and Unilateral Mechanics. Kluwer Academic Publishers, Boston (2003)

14. Hiriart-Urruty, J.B., Lemaréchal, C.: Fundamentals of Convex Analysis. Springer (1993)

15. Hlavacek, I., Lovisek, J.: Semi-coercive variational inequalities with uncertain input data. Applications to shallow shells. Mat. Mod. Meth. Appl. Sci. 15(2), 273-299 (2005)

16. Kinderlehere, D., Stampacchia, G.: An Introduction to Variational Inequalities and their Applications. Academic Press, New York (1980)

17. Lions, J.L.: Quelques méthodes de résolution des problèmes aux limites non-linéaires. Dunod (1969)

18. Lions, J.L., Stampacchia, G.: Variational inequalities. Comm. Pure Appl. Math. 20, 493-519 (1967)

19. Panagiotopoulos, P.D.: Inequality Problems in Mechanics and Applications, Convex and Nonconvex Energy Functions. Birkhaüser, Basel (1985)

20. Rockafellar, R.T.: Convex Analysis. Princeton, University Press, Princeton, NJ (1970)

21. Tomarelli, F.: Noncoercive Variational Inequalities for Pseudomonotone Operators. Preprint (1993)

22. Vy, L.K., Ang, D.D.: Frictional contact of an elastic body with a rigid support. Nonlin. Anal. TMA 25(24), 339-343 (1995) 